\begin{tabular}{c} 
International Journal of Advanced Astronomy, 5(2)(2017) $117-123$ \\
International Journal of Advanced Astronomy \\
SPC \\
Website $\begin{array}{c}\text { www.sciencepubco.com/index. } p h p / I J A A \\
\text { doi:10.14419/ijaa. } v 5 i 2.8168 \\
\text { Research paper }\end{array}$ \\
\hline
\end{tabular}

\title{
FLRW solution in bimetric theory of gravitation
}

\author{
M. S. Borkar, A. Ameen* \\ Post Graduate Department of Mathematics, Mahatma Jyotiba Phule Educational Campus, R. T. M. Nagpur University, Nagpur- 440 033 \\ (India) \\ Department of Mathematics, Jawaharlal Darda Institute of Engineering and Technology, Yavatmal-445 001(India) \\ *Corresponding author E-mail:ameen_ashaikh@rediffmail.com
}

\begin{abstract}
We have derived FLRW line-element in Bimetric Theory of Gravitation (BTG) by solving Rosen's field equations, and it is concluded that the geometry of our model in BTG is agreed with the geometry of FLRW model in GR. It is also realized that for the large value of $t$, the deceleration parameter $q$ in our model admits the value $(-1)$ which is close to the value $(\approx-0.77)$ at present epoch predicted by the observations of [44-48].This This shows that our FLRW model in BTG is found to be in an accelerating phase at present epoch which is not the case in GR. Other geometrical and physical aspects to the model are also studied.
\end{abstract}

Keywords: FLRW Model; Bimetric Theory of Gravitation; Cosmology; Isotropy.

\section{Introduction}

The spherical symmetry has its own importance in General Relativity (GR) theory by virtue of its comparative simplicity. Many important space-times such as Schwarzschild (exterior and interior) solutions, the Robertson-Walker models of expanding universe, etc. are all spherically symmetric. The large-scale distribution of galaxies in our universe shows that the matter distribution is satisfactorily described by perfect fluid. The whole universe is spherically symmetric in nature and therefore, it is important to study the spherically symmetric cosmological models of the universe. Scientific cosmology really began, when Albert Einstein [1] published his field equations of GR. The current cosmological models are based on GR because this theory is what produces at present the best agreement for large-scale behavior of the universe. There are two types of cosmological models: Static cosmological models and Non-static cosmological models. The static cosmological models are described by Einstein and de- Sitter. Einstein universe is full of matter, and it does not allow any motion while the deSitter universe is empty and it allows motion. When Einstein derived his static model, the universe was assumed to be static in nature. But later on, Edwin Hubble [2] and Hubble and Humason [3] have shown that the universe is not static, but it is expanding. Since the static models do not really represent the visible universe. Therefore, in order to describe the behavior of visible universe, it was essential to study the non-static models of the universe.

Friedman [4-5] concluded that if the Einstein's theory of GR was correct then our universe must either expand or contract. He was the first who proposed the non-static model of expanding universe. Robertson [6] made a simple assumption that on the large scale, the universe is homogeneous and isotropic for obtaining a nonstatic model of the universe. The work on non-static cosmological models which was initiated by Friedman [4-5], further it was carried out independently by Lemaitre [7] and Walker [8].
The model obtained by them is called Friedman- Lemaitre- Robertson- Walker (FLRW) metric which rules the evolution of the universe and it is expressed in the form

$d s^{2}=-\frac{e^{g(t)}}{\left[1+\left(k r^{2} / 4 a^{2}\right)\right]^{2}}\left[\mathrm{dr}^{2}+r^{2} d \theta^{2}+r^{2} \sin ^{2} \theta d \varphi^{2}\right]+d t^{2}$,

where $k$ is the curvature constant which can assume the values $-1,0$ and +1 respectively for open, flat and closed models of the universe. This metric is spherically symmetric giving an exact solution of Einstein's field equations of GR and describes a homogeneous, isotropic expanding or contracting universe. It is the standard model of modern cosmology. FLRW model is important and has much physical significance in the study of the universe and therefore many researchers [9-13] have examined the geometrical and physical behavior of it in GR and also in other modified gravitational theories and exposed the nature of the universe. Still findings are going on about the nature of FLRW model in modified gravitational theories, since FLRW model shows that our universe is homogeneous and isotropic in nature and it is supported by various recent cosmological observations [14-20].

One of the successful modified theory to GR is Rosen's Bimetric Theory of Gravitation (BTG) [21-23], since it is free from singularities and obeys the principle of covariance and principle of equivalence of GR and it is based on two metrics: One is the Riemannian metric with metric potentials $g_{i j}$ and other is flat metric $\gamma_{i j}$ attached at every point of space-time. The field equations of this BTG are

$N_{i}^{j}-\frac{1}{2} N \quad \delta_{i}^{j}=-T_{i}^{j}$,

where

$N_{i}^{j}=\frac{1}{2} \gamma^{\alpha \beta}\left(g^{s j} g_{s i \backslash \alpha}\right)_{\mid \beta}$, 
is the Rosen's Ricci-tensor and $N=g_{i j} N^{i j}$ is the Rosen's scalar. Here and hereafter the vertical bar (I ) stands for $\gamma$-covariant differentiation with $g=\operatorname{det}\left(g_{i j}\right)$ and $\gamma=\operatorname{det}\left(\gamma_{i j}\right)$.

Many researchers have been developing the cosmological models of the universe in this BTG and trying to evaluate their geometrical and physical properties. Some of them are Rosen [21-23], Karade [24], Isrelit, [25], Yilmaz [26], Reddy and Venkateshwara Rao [27], Katore and Rane [28], Khadekar and Tade [29], Borkaret al. [30-39], Borkar and Ameen [40-42], Gaikwad et al. [43] etc. In this Paper, we have taken-up the study of FLRW model in BTG and obtained the solutions of Rosen's field equations (2) and (3) in regards with the FLRW metric. Some geometrical and physical aspects of the FLRW model have been studied in BTG. It is realized that the geometry of our derived model in BTG is matched with the geometry of FLRW model in GR. Further, it is seen that the value of the deceleration parameter $q$ is found as (-1) at present epoch which is very near to the present observed value $(\approx-0.77)$ of the deceleration parameter at late times as per recent observations of [44-48].

\section{Metric and field equations}

The spherically symmetric FLRW metric is

$$
d s^{2}=-e^{f+g}\left[\mathrm{dr}^{2}+r^{2} d \theta^{2}+r^{2} \sin ^{2} \theta d \varphi^{2}\right]+d t^{2},
$$

where $f$ and $g$ are the functions of $r$ and $t$ respectively.

The background flat metric corresponding to the metric (4) is given by

$d \eta^{2}=-e^{f}\left[\mathrm{dr}^{2}+r^{2} d \theta^{2}+r^{2} \sin ^{2} \theta d \varphi^{2}\right]+d t^{2}$.

The energy momentum tensor $T_{i}^{j}$ for a perfect fluid distribution is

$T_{i}^{j}=(p+\rho) v_{i} v^{j}-p g_{i}^{j}$,

where $p$ is the isotropic pressure, $\rho$ is the energy density and $v^{i}$ is the four velocity vector with magnitude $g_{i j} v^{i} v^{j}=1$.

The co-moving co-ordinates is to be assumed. So that

$v^{1}=v^{2}=v^{3}=0$ and $v^{4}=1$.

The components of energy momentum tensor $T_{i}{ }^{j}$ for a perfect fluid distribution from the equations (6) and (7) are

$T_{1}^{1}=T_{2}^{2}=T_{3}^{3}=-p, T_{4}^{4}=\rho$

Simplifying the Rosen's field equations (2) and (3) for the metrics (4) and (5) with the help of equation (8), we arrived at the following differential equations.

$\frac{1}{4} \ddot{g}-\frac{1}{4} e^{-f} f^{\prime \prime}+\frac{1}{r^{2}} e^{-f}+\frac{1}{2 r^{2}} e^{-f} \operatorname{cosec}^{2} \theta=-p$,

$\frac{1}{4} \ddot{g}-\frac{1}{4} e^{-f} f^{\prime \prime}+\frac{1}{2 r^{2}} e^{-f} \operatorname{cosec}^{2} \theta=-p$,

$\frac{1}{4} \ddot{g}-\frac{1}{4} e^{-f} f^{\prime \prime}-\frac{1}{2 r^{2}} e^{-f} \operatorname{cosec}^{2} \theta=-p$,

$\frac{3}{4} \ddot{g}-\frac{3}{4} e^{-f} f^{\prime \prime}+\frac{1}{r^{2}} e^{-f}+\frac{1}{2 r^{2}} e^{-f} \operatorname{cosec}^{2} \theta=\rho$,

where overhead dots and primes denote differentiation with respect to $t$ and $r$ respectively.
From the above differential equations (9) and (10), we write

$$
\frac{e^{-f}}{r^{2}}=0
$$

With this equation (13), the above differential equations (9-12) reduce in two equations

$\frac{1}{4} \ddot{g}-\frac{1}{4} e^{-f} f^{\prime \prime}=-p$,

$\frac{3}{4} \ddot{g}-\frac{3}{4} e^{-f} f^{\prime \prime}=\rho$.

\section{Solution of the field equations}

On large scale, the universe is homogeneous and isotropic in nature with the distribution of perfect fluid and therefore, we are using the isotropic condition in our study to evaluate the model. We are going to solve these two differential equations (14) and (15) which are in three un-knowns $f, p$ and $\rho$. Therefore, in order to have a unique solution, we require only one extra condition to solve the differential equations (14) and (15). We proceed the matter with the assumption $T_{1}^{1}=-T_{4}^{4}$ and then the equations (14) and (15) using equation (8) yield

$\ddot{g}=e^{-f} f^{\prime \prime}$.

In this equation (16), the right-hand side is a function of $r$ only and left-hand side is a function of $t$ only and therefore each side can be taken as a constant (say) $a_{1}$. Thus, we write

$\ddot{g}=e^{-f} f^{\prime \prime}=a_{1}($ Constant $)$.

So that we have

$\ddot{g}=a_{1}$,

and

$e^{-f} f^{\prime \prime}=a_{1}$.

We are solving the differential equation (19). For this, we substitute

$e^{-f}=F(r)$.

We write

$F F^{\prime \prime}-F^{\prime 2}-a_{1} F=0$

Now substituting $F^{\prime}=\left(\frac{d F}{d r}\right)=m$

and

$F^{\prime \prime}=\left(\frac{d^{2} F}{d r^{2}}\right)=m\left(\frac{d m}{d F}\right)$

The above differential equation (21) reduces to

$m \frac{d m}{d F}-\frac{m^{2}}{F}=a_{1}$,

which can be reduced further as 
$\frac{d n}{d F}-\frac{2}{F} n=2 a_{1}$

With the value $m^{2}=n$. the solution of this equation (23) is given by

$\frac{n}{F^{2}}=c-\frac{2 a_{1}}{F}$

OR

$\left(\frac{d F}{d r}\right)^{2}=c F^{2}-2 a_{1} F$

OR

$[F(F-1)]^{-\frac{1}{2}} d F=\sqrt{c} d r$

where $c$ is the constant of integration and it is chosen as $c=2 a_{1}$. To solve the above equation (25), we are choosing $F$ as $F=\cosh ^{2} \eta$, so that

$d F=2 \cosh \eta \sinh \eta d \eta$,

and the equation (25) gives

$d \eta=\frac{\sqrt{c}}{2} d r$,

which after integration, yields

$\eta=\frac{\sqrt{c}}{2} r+c_{1}$

OR

$\cosh ^{-1}(\sqrt{F})=\frac{\sqrt{c}}{2} r+c_{1}$

OR

$F=\cosh ^{2}\left(\frac{\sqrt{c}}{2} \mathrm{r}+\mathrm{c}_{1}\right)$

where $c_{1}$ is the constant of integration. For simplicity, selecting $c_{1}=0$. so that

$e^{f}=\operatorname{sech}^{2}\left(\frac{\sqrt{c}}{2} r\right)=\frac{1}{\left[1+\left(c r^{2} / 8\right)\right]^{2}}$.

Thus, the form of FLRW line-element in Bimetric Theory of Gravitation is

$d s^{2}=-\frac{e^{g(t)}}{\left[1+\left(c r^{2} / 8\right)\right]^{2}}\left[\mathrm{dr}^{2}+r^{2} d \theta^{2}+r^{2} \sin ^{2} \theta d \varphi^{2}\right]+d t^{2}$.

It is interesting to evaluate the geometrical and physical behavior of this FLRW model in BTG. On comparing this line-element (28) with FLRW model in GR, it is seen that our model (28) agreed with the FLRW model of GR with the value of constant $c=2 k / a^{2}$ in which $k=1,0,-1$ having the usual meaning in FLRW model in GR corresponding to closed, flat and open universe. Thus, it is observed that the geometrical features of FLRW model in Bimetric theory of gravitation matched with that of the geometrical features in GR with the value of constant $c=2 k / a^{2}$ and there is no any new behavior found in regards with its geometry in the evolution.

\section{Properties of our FLRW model}

We are going to evaluate some more geometrical and physical properties of this model in Bimetric gravitational theory.

\subsection{Co-ordinate transformations}

In regards with different co-ordinate transformations, we are trying to see the nature of the model.

Under the transformations,

$x=r \sin \theta \cos \varphi, \quad y=r \sin \theta \sin \varphi, \quad \mathrm{z}=\mathrm{r} \cos \theta$,

our model (28) is

$d s^{2}=-\frac{e^{g(t)}}{\left[1+\left(c r^{2} / 8\right)\right]^{2}}\left(\mathrm{dx}^{2}+d y^{2}+d z^{2}\right)+d t^{2}$

and it is the spatial isotropy of three space.

With the co-ordinates transformation

$r^{\prime}=\frac{r}{\left[1+\left(c r^{2} / 8\right)\right]}, \theta, \varphi, t$

our FLRW model (28) having the form

$d s^{2}=-e^{g(t)}\left[\frac{d r^{2}}{\left[1-\left(c r^{2} / 2\right)\right]}+r^{2} d \theta^{2}+r^{2} \sin ^{2} \theta d \varphi^{2}\right]+d t^{2}$,

and it is non-static but its geometry matched with the static Einstein universe with radius $c / 2$. This line-element (30) also represents three-dimensional spherical surface

$\left(x^{1}\right)^{2}+\left(x^{2}\right)^{2}+\left(x^{3}\right)^{2}+\left(x^{4}\right)^{2}=a^{2}$ embedded in the four- dimensional

Euclidean space $\left(x^{1}, x^{2}, x^{3}, x^{4}\right)$.

With Riemannian curvature $k=c / 2$ (it is $1 / a^{2}$ in GR). $c>0$ gives the spherical space of positive curvature and in this case our model (30) is closed. The quantity $c<0$ yields a pseudo spherical space of negative curvature with open model (30). For $c=0$, we have flat Euclidean space and the curvature of our model (30) is zero.

Let us calculate the radius of universe $R(t)$ in our model (30). With the co-ordinates $r, \theta, \varphi$, we can find the radius of universe $R(t)$ with respect to the constant $c$ in model (30). The circumference of the circle is $2 \pi r$ and surface of sphere is $4 \pi r^{2}$. So that the radius $R$ of sphere can be taken as

$R=\int_{0}^{r} \frac{d r}{\sqrt{1-\left(c r^{2} / 2\right)}}$

and its value is

$R=\sqrt{\frac{2}{c}} \sin ^{-1}\left[\frac{r}{\sqrt{2 / c}}\right]$

which is greater than $r$. So that the ratio of circumference with radius is $2 \pi r / R<2 \pi$.

Assuming the radial co-ordinate $r$ in the model (30) as $r=(\sqrt{2 / c}) \sin \psi$, where the angle $\psi(0 \leq \psi \leq \pi)$. With the value of 
radial co-ordinate $r$, we have $d r=(\sqrt{2 / c}) \cos \psi d \psi$ and $1-\left(c r^{2} / 2\right)=\left(1-\sin ^{2} \psi\right)=\cos ^{2} \psi$.

With these substitutions, our line-element (30) reduces to

$d s^{2}=-R^{2}(t)\left[d \psi^{2}+\sin ^{2} \psi\left(d \theta^{2}+\sin ^{2} \theta d \varphi^{2}\right)\right]+d t^{2}$,

in which

$R^{2}(t)=(2 / c) e^{g(t)}$,

is called the radius of the universe and it is an infinite, when $c=0$. The distance of the point on the surface from the origin is $\left(\sqrt{2 / c} \mathrm{e}^{\mathrm{g} / 2}\right) \psi$ and the surface of the sphere is $(8 \pi / c) \mathrm{e}^{\mathrm{g}(t)} \sin ^{2} \psi$. It is important to note that the surface of the sphere increases as we move away from the origin becomes maximum at $\psi=\pi / 2$ at a distance $(\sqrt{2 / c}) \pi / 2$ and then it starts decreasing and attains minimum value at $(\sqrt{2 / c}) \psi[=(\sqrt{2 / c}) \pi]$. With this real $R(t)$, total proper spatial volume of the model at any time $t$ has been calculated as

$V=2 \pi^{2}\left(\frac{2 \mathrm{e}^{g(t)}}{c}\right)^{3 / 2}$

\subsection{Motion of the particles in the model}

We consider the behavior of free particles in our model (28). In reference to the principles of relativistic mechanics, the motion of free particles is given by the geodesic equation

$\frac{d^{2} x^{\sigma}}{d s^{2}}+\Gamma_{\mu \nu}^{\sigma} \frac{d x^{\mu}}{d s} \frac{d x^{v}}{d s}=0$.

We are considering two situations, first is particle is at rest and second is particle moving along the radial direction. In the first situation means for rest particle, we write

$\frac{d r}{d s}=\frac{d \theta}{d s}=\frac{d \varphi}{d s}=0$

Our model (28) gives

$$
1=-\frac{e^{g(t)}}{\left[1+\left(\mathrm{cr}^{2} / 8\right)\right]^{2}}\left\{\left(\frac{d r}{d s}\right)^{2}+r^{2}\left[\left(\frac{d \theta}{d s}\right)^{2}+\sin ^{2} \theta\left(\frac{d \varphi}{d s}\right)^{2}\right]\right\}+\left(\frac{d t}{d s}\right)^{2},
$$

and with the initial condition (34), the above equation (35) gives

$$
\left(\frac{d t}{d s}\right)^{2}=1
$$

and with the conditions (34) and (36), the geodesic equation (33) yields

$\frac{d^{2} r}{d s^{2}}=0=\frac{d^{2} \theta}{d s^{2}}=\frac{d^{2} \varphi}{d s^{2}}=\frac{d^{2} t}{d s^{2}}$

Thus, all components of acceleration of a particle which is at rest is zero. So its acceleration vanishes and hence it would remain permanently at rest with respect to spatial co-ordinates $r, \theta, \varphi$ and would experience no gravitational acceleration. Our observations are same with those of rest particle in GR.

In the second situation, suppose a particle is moving along the radial direction. So that it has no velocity component along $\theta$ and $\varphi$ directions. Therefore, we have $\frac{d \theta}{d s}=\frac{d \varphi}{d s}=0$,

and hence the geodesic equation (33) yields

$\frac{d^{2} \theta}{d s^{2}}+\Gamma_{11}^{2}\left(\frac{d r}{d s}\right)^{2}+2 \Gamma_{14}^{2}\left(\frac{d r}{d s}\right)\left(\frac{d t}{d s}\right)+\Gamma_{4(}^{2}\left(\frac{d t}{d s}\right)^{2}=0$,

and

$\frac{d^{2} \varphi}{d s^{2}}+\Gamma_{11}^{3}\left(\frac{d r}{d s}\right)^{2}+2 \Gamma_{14}^{3}\left(\frac{d r}{d s}\right)\left(\frac{d t}{d s}\right)+\Gamma_{44}^{3}\left(\frac{d t}{d s}\right)^{2}=0$,

and they give

$\frac{d^{2} \theta}{d s^{2}}=0$ and $\frac{d^{2} \varphi}{d s^{2}}=0$.

From these equations, it is clear that the acceleration of the particle along $\theta$ and $\varphi$ directions is zero. Thus, the particle has acceleration only along the radial direction. From this, we have concluded that the particle continues to travel in the radial direction and this is due to spatial isotropy of the model.

Now let us obtain the general motion of the particle in the model. Suppose the particle has an initial velocity. For the time variable $t$ the geodesic equation (33) takes the form

$\frac{d^{2} t}{d s^{2}}+\Gamma_{11}^{4}\left(\frac{\mathrm{dr}}{\mathrm{ds}}\right)^{2}+\Gamma_{22}^{4}\left(\frac{\mathrm{d} \theta}{\mathrm{ds}}\right)^{2}+\Gamma_{33}^{4}\left(\frac{\mathrm{d} \varphi}{\mathrm{ds}}\right)^{2}+\Gamma_{44}^{4}\left(\frac{\mathrm{d} t}{\mathrm{ds}}\right)^{2}=0$,

which gives

$$
\frac{\mathrm{d}^{2} t}{d s^{2}}+\frac{e^{g(t)}}{2\left[1+\left(c r^{2} / 8\right)\right]^{2}} \frac{d g}{d t}\left\{\left(\frac{d r}{d s}\right)^{2}+r^{2}\left[\left(\frac{d \theta}{d s}\right)^{2}+\sin ^{2} \theta\left(\frac{d \varphi}{d s}\right)^{2}\right]\right\}=0 .
$$

From equations (35) and (37), we get

$\frac{\mathrm{d}^{2} t}{d s^{2}}+\frac{1}{2} \frac{d g}{d t}\left[\left(\frac{d t}{d s}\right)^{2}-1\right]=0$.

On using the substitution $y=\left(\left(\frac{d t}{d s}\right)^{2}-1\right)$, the equation (38)can be re-written as

$\frac{d y}{d t}+y \frac{d g}{d t}=0$,

which on integration gives

$y=A e^{-g(t)}$

OR

$\left(\frac{d t}{d s}\right)^{2}-1=A e^{-g(t)}$

where $A$ is constant of integration.

From equations (35) and (39), we get

$\frac{e^{g(t)}}{\left[1+\left(\mathrm{cr}^{2} / 8\right)\right]^{2}}\left(\frac{d t}{d s}\right)^{2}\left\{\begin{array}{l}\left(\frac{d r}{d t}\right)^{2} \\ +r^{2}\left[\left(\frac{d \theta}{d t}\right)^{2}+\sin ^{2} \theta\left(\frac{d \varphi}{d s}\right)^{2}\right]\end{array}\right\}=A e^{-g(t)}$. 
From the line-element (28), we get

$$
\frac{d s^{2}}{d t^{2}}=1-\frac{e^{g(t)}}{\left[1+\left(\mathrm{cr}^{2} / 8\right)\right]^{2}}\left\{\left(\frac{d r}{d t}\right)^{2}+r^{2}\left[\left(\frac{d \theta}{d t}\right)^{2}+\sin ^{2} \theta\left(\frac{d \varphi}{d s}\right)^{2}\right]\right\},
$$

which on comparing with the motion of particles

$\frac{d s^{2}}{d t^{2}}=1-\left(u^{2} / v^{2}\right)$

(where $v$ is the velocity of light and $u$ is the velocity of the particle measured by an observer at rest with respect to the coordinates $r, \theta, \varphi$.$) , we get$

$u^{2}=\frac{v^{2} e^{g(t)}}{\left[1+\left(\mathrm{cr}^{2} / 8\right)\right]^{2}}\left\{\left(\frac{d r}{d t}\right)^{2}+r^{2}\left[\left(\frac{d \theta}{d t}\right)^{2}+\sin ^{2} \theta\left(\frac{d \varphi}{d s}\right)^{2}\right]\right\}$.

From equations (39), (40) and (43), we write the velocity of particle $\mathrm{u}$ as

$u^{2}=\frac{v^{2} A}{\left[e^{g(t)}+A\right]}$.

From equation (44), it is obvious that in the expanding universe, the function $g(t)$ will increase with time $t$ and the velocities of free particles will decrease with time and in the contracting universe $g(t)$ will decrease with time and the velocities will increase with time. Thus, it is realized that the motion of particle at rest, along radial direction and general motion of test particle in our FLRW model (28) in Bimetric gravity follows the same path as that of the motions in FLRW model in GR and there is no any new geometrical properties contributing in the motion of test particle.

\subsection{Motion of the light rays in the model}

For a ray of light, we have

$d s=0$,

and in view of our model (28), this equation $d s=0$ for light rays gives

$\frac{e^{g(t)}}{\left[1+\left(\mathrm{cr}^{2} / 8\right)\right]^{2}}\left[\left(\frac{d r}{d t}\right)^{2}+r^{2}\left(\frac{d \theta}{d t}\right)^{2}+r^{2} \sin ^{2} \theta\left(\frac{d \varphi}{d t}\right)^{2}\right]=1$.

From this equation (46) and equation (42), we obtain the relation between velocity of light $v$ and particle velocity $u$ as

$u=v$

From which we conclude that the velocity of light is same as that of the velocity of particle.

Let us suppose that a light ray moves initially in the radial direction. So its velocity along $\theta$ and $\varphi$ directions will be zero. Thus, we have

$$
\frac{d \theta}{d t}=\frac{d \varphi}{d t}=0
$$

The equation (46), with the equation (47), yields

$$
\frac{d r}{d t}= \pm e^{-\frac{g(t)}{2}}\left(1+\left(\frac{c r^{2}}{8}\right)\right)
$$

In the equation (48), + sign corresponds to a ray travelling away from the origin and - sign represents a ray which travels towards the origin. Let us assume that the light ray starts travelling from the origin at time $t_{1}$ and reaches the point $r$ at time $t_{2}$. then from equation (48), we write

$\int_{0}^{r} \frac{d r}{\left[1+\left(c r^{2} / 8\right)\right]}=\int_{t_{1}}^{t_{2}} e^{-\frac{g(t)}{2}} d t$

which gives

$$
\frac{2^{3 / 2}}{\sqrt{c}} \tan ^{-1}\left(\frac{r \sqrt{c}}{2^{3 / 2}}\right)=\int_{t_{1}}^{t_{2}} e^{-\frac{g}{2}} d t
$$

In regards with the expression for light trajectory, the Tolman [49] has assumed the linear expression for $g(t)$, but in our FLRW model (28), we have $\ddot{g}=a_{1}$ (constant) which provides $g(t)$ as a quadratic function of time $t$. Therefore, on the line of Tolman [49], we are taking in particular $a_{1}=0$. i.e., the linear expression for the function $g(t)$. Thus, we assume $\ddot{g}=0$ that gives $g(t)=\alpha t+\beta$, where $\alpha, \beta$ are constants of integration. In view of this, we have solved the equation (50). We choose $\alpha=2 b$, and $\beta=0$ then the expression for $g(t)$ is given by

$g(t)=2 b t$

Using equation (51) in equation (50), we get

$r=\frac{2^{3 / 2}}{\sqrt{c}} \tan \left[\frac{\left(e^{-b t_{1}}-e^{-b t_{2}}\right) \sqrt{c}}{2^{3 / 2} b}\right]$.

The equation (52) is the equation of light trajectory for our model (28). We observed the light trajectory given by (52) in particular for $\ddot{g}=0$ means $a_{1}=0$ not in general case for $\ddot{g}=a_{1} \neq 0$ in our model.

\subsection{The red shift}

Our model (28) can be expressed as

$d s^{2}=d t^{2}-\frac{c R^{2}(t)}{2\left[1+\left(c r^{2} / 8\right)\right]^{2}}\left[d r^{2}+r^{2}\left(d \theta^{2}+\sin ^{2} \theta d \varphi^{2}\right)\right]$,

where $R(t)$ is the radius of universe and in our derived model its value is

$R(t)=\sqrt{2} \mathrm{c}^{-\frac{1}{2}} e^{\frac{g(t)}{2}}$.

We take a distant radiating source as a galaxy $G$ which can be considered as a particle with co-ordinates $\left(r_{1}, \theta_{1}, \varphi_{1}\right)$. Then assume that a light ray emitted by $G$ is observed at the origin $O$ so that it is moving along the radial direction towards $O$. Then equation (53) yields

$\frac{d t}{R(t)}=\frac{\sqrt{c} d r}{\sqrt{2}\left[1+\left(c r^{2} / 8\right)\right]}$.

Now we suppose that the light ray leaves $G$ at time $t_{1}$ and reaches $O$ at time $t_{0}$ such that $t_{0}>t_{1}$. Then above equation (55) gives

$\int_{t_{1}}^{t_{0}} \frac{d t}{R(t)}=\int_{r_{1}}^{0} \frac{\sqrt{c} d r}{\sqrt{2}\left[1+\left(c r^{2} / 8\right)\right]}=f\left(r_{1}\right)$, (say). 
Next we suppose that the light ray is emitted through $G$ at time $t_{1}+\delta t_{1}$ and arrived at $O$ at time $t_{0}+\delta t_{0}$. Then again this leads to similar equation (56), which is given by

i.e. $\int_{t_{1}+\delta_{t_{1}}}^{t_{0}+\delta_{t_{0}}} \frac{d t}{R(t)}=f\left(r_{1}\right)$

which further can be written as

$\int_{t_{1}+\delta_{1}}^{t_{1}} \frac{d t}{R(t)}+\int_{t_{1}}^{t_{0}} \frac{d t}{R(t)}+\int_{t_{0}}^{t_{0}+\delta_{t_{0}}} \frac{d t}{R(t)}=f\left(r_{1}\right)$

The result from elementary calculus gives

$\int_{t}^{+t \delta t} F(t) d t=F(t) \delta t$,

where $F(t)$ varies very little over a small interval $\delta t$.

From equations (56-58), we get

$\frac{\delta t_{1}}{\delta t_{0}}=\frac{R\left(t_{1}\right)}{R\left(t_{0}\right)}$

Let $\lambda_{1}, v_{1}$ be the wave length and frequency respectively of the light ray emitted from $G$ at time $t_{1}$, and $\lambda_{0}, v_{0}$ denote the corresponding quantities of the same light ray observed at $O$. Then we have

$\frac{v_{0}}{v_{1}}=\frac{\lambda_{1}}{\lambda_{0}}=\frac{\delta t_{1}}{\delta t_{0}}$

and with the relation (60), we write

$\frac{\lambda_{1}}{\lambda_{0}}=\frac{R\left(t_{1}\right)}{R\left(t_{0}\right)}$.

A red shift parameter $z$ is defined as

$z=\left(\lambda_{0}-\lambda_{1}\right) / \lambda_{1}$

which with the relation (62), yields

$z=\left[R\left(t_{0}\right) / R\left(t_{1}\right)\right]-1$.

From equation (64), we opined the red shift if $z>0$ means $\lambda_{0}>\lambda_{1}$ and we arrived at blue shift if $z<0$ means $\lambda_{0}<\lambda_{1}$. For expanding universe the radius of universe $R(t)$ is monotonic increasing function of time t, so that $R\left(t_{0}\right)>R\left(t_{1}\right), t_{0}>t_{1}$, when the universe is expanding and in this situation we get a red shift. For the contracting universe, the radius of the universe $R(t)$ is monotonic decreasing function of time $t$, and then we get $R\left(t_{0}\right)<R\left(t_{1}\right)$ for contracting universe and the relation (64) gives the blue shift. These are observations which have been observed in our model (28) in BTG regarding red shift and blue shift which are resembled to the results obtained in GR.

\subsection{Deceleration parameter and Hubble's parameter}

The deceleration parameter $q$ is given by

$q=-\frac{R \ddot{\mathrm{R}}}{\dot{\mathrm{R}}^{2}}$

and in our model (28), it has value $q=-1-\frac{c}{\left(b_{1}+c t / 2\right)^{2}}$,

where $b_{1}$ is the constant of integration. For the large value of $t$, the deceleration parameter $q$ admits the value $(-1)$ which is close to the value $(\approx-0.77)$ at present epoch predicted by the observations of [44-48].Thus, our FLRW model (28) in Bimetric theory of gravitation is found to be in an accelerating phase for large value of $t$. The Hubble's parameter $H$ for our model (28) is given by

$H=\dot{R} / R$

and in our model it has value

$H=\frac{1}{2}\left[\frac{c t}{2}+b_{1}\right]$.

The Hubble's parameter $H$ is directly proportional to time $t$ in our model whereas it is inversely proportional to time $t$ occurring in the most of the works of [50-52] etc. It is increasing continuously with time so that the rate of expansion in the model is continuously increasing. In particular, for $a_{1}=0$ we get

$c=0, q=-1$ and $H=b_{1} / 2$,

from which, it is clear that the model is flat which has an accelerating phase always and expanding with constant rate of expansion for $a_{1}=0$.

\section{Summary}

- The FLRW model has been derived in Bimetric theory of gravitation by solving the Rosen's field equations. It is seen that our model in BTG is agreed with FLRW model in GR with the value of constant $c=2 k / a^{2}$, where $k=1,0,-1$ corresponding to closed, flat and open universe.

- It is observed that the geometry of FLRW model in BTG is matched with that of the geometry of the FLRW model in GR.

- In regards with different co-ordinate transformations, the geometrical features of our model in BTG are same as that of geometrical features of FLRW model in GR.

- It is realized that the motion of particle at rest, along radial direction and general motion of test particle in our model in BTG follows the same path as that of the motions in FLRW model in GR.

- It is observed that in our BTG model, the light trajectory follows the same path as that of FLRW model in GR, in particular for $a_{1}=0$ and not in general case for non-zero $a_{1}$. For the case $a_{1}=0$ we get $c=0, q=-1$ and $H=b_{1} / 2$ which shows that our model is flat, has an accelerating phase always and expanding with constant rate of expansion in the case for $a_{1}=0$.

- We have observed the red shift and the blue shift of the spectrum in our model in BTG which are resembled to the results obtained in GR.

- It is realized that our model in BTG has an accelerating expansion at late times which is not in GR. In GR, FLRW model may or may not be accelerating at late times.

- The rate of expansion in the model is continuously increasing. The Hubble's Parameter $H$ is a function of time $t$ which is directly proportional to $t$ in our model whereas it is inversely proportional to time $t$ in most of the results of [5052] etc. We observed that the rate of expansion in our model is continuously increasing with time $t$. 


\section{Conclusion}

In this paper, the FLRW line-element has been derived in Bimetric theory of gravitation by solving Rosen's field equations, and it is found that the geometry of our model in BTG is similar to the geometry of FLRW model in GR. It is observed that for the particular value of constant $a_{1}=0$ appearing in the expression for $g(t)$ in our model in BTG, our derived model becomes flat and it is always acceleratingly expanding with constant rate of expansion, which is not in GR. Other geometrical and physical aspects of the model have been carried out and it is realized that our model is having the similar features as that of FLRW model in GR in relation with co-ordinate transformations, motion of test particle at rest, along radial direction, general motion and light rays properties and red shift.

\section{References}

[1] Einstein, A. (1915), Sitzungsber. Preus. Akad. Wiss. Berlin (Math. Phys.) 778. https://doi.org/10.1073/pnas.15.3.168.

[2] Hubble, E. (1929), Proc. Natl. Acad. Sci. U.S.A., 15:168.https://doi.org/10.1073/pnas.15.3.168.

[3] Hubble, E.andHumason, M. L. (1931), Astrophysical Journal, 74: 43. https://doi.org/10.1086/143323

[4] Friedman, A. (1922), Z. Phy., 10: 377.https://doi.org/10.1007/BF01332580.

[5] Friedman, A. (1924), ZeitschriftfürPhysik, 21(1): 326.https://doi.org/10.1007/BF01328280.

[6] Robertson, H. P. (1935), Astrophysical Journal 82: 284. https://doi.org/10.1086/143681.

[7] Lemaitre, G (1927), Monthly Notices of the Royal Astronomical Society 91: 483.https://doi.org/10.1093/mnras/91.5.483.

[8] Walker, A. G. (1937), Proc. of the London Math. Soc., 42: 90.https://doi.org/10.1112/plms/s2-42.1.90.

[9] Burd, A. B. and Barrow, J.D. (1988), Nucl. Phys. B, 308: 923.https://doi.org/10.1016/0550-3213(88)90135-6.

[10] Wald, R. (1983), Phys. Rev. D, 28: 2118.https://doi.org/10.1103/PhysRevD.28.2118.

[11] Barrow, J.D. (1987), Phys. Lett. B, 187: 12.https://doi.org/10.1016/0370-2693(87)90063-3.

[12] Ellis, G.F.R. and Madsen, M.S. (1991), Class. Quantum Gravity, 8: 667.https://doi.org/10.1088/0264-9381/8/4/012.

[13] Heusler, M. (1991), Phys. Lett. B, 253: 33.https://doi.org/10.1016/0370-2693(91)91359-4.

[14] Spergel, D. N., et al.(2003), Astrophys. J. Suppl. 148: 175.https://doi.org/10.1086/377226.

[15] Spergel, D. N., et al. (2007), Astrophys. J. Suppl. 170: 377. https://doi.org/10.1086/513700.

[16] Komatsu, E., et al.(2009), Astrophys. J. Suppl. 180: 330.https://doi.org/10.1088/0067-0049/180/2/330.

[17] Komatsu, E., et al.(2011), Astrophys.J. Suppl., 192: 18.https://doi.org/10.1088/0067-0049/192/2/18.

[18] Hinshaw, G., et al.(2013), Astrophys. J. Suppl., 208: 19.https://doi.org/10.1088/0067-0049/208/2/19.

[19] Ade, P. A. R., et al. (2013a), arXiv: 1303.5076.

[20] Ade, P. A. R.et al. (2013b), arXiv: 1303.5082.

[21] Rosen, N. (1973), Gen. Rel. and Grav, 4 (6): 435.https://doi.org/10.1007/BF01215403.

[22] Rosen, N. (1974), Annls of Physics. 84: 455.https://doi.org/10.1016/0003-4916(74)90311-X.

[23] Rosen, N. (1977), Plenum Press, London. 273.

[24] Karade, T. M. (1980), Indian J. Pure-appl. Math., 11(9): 1202.

[25] Israelit, M. (1981), Gen. Rel. Grav., 13(7): 681. https://doi.org/10.1007/BF00759411.

[26] Yilmaz, H. (1975), Gen. Relativ. Gravit, 6: 269.https://doi.org/10.1007/BF00751571.

[27] Reddy, D.R.K. and Venkateswara Rao, N. (1998), Astrophys. Space Sci., 257: 293.https://doi.org/10.1023/A:1001166619709.

[28] Katore, S.D. and Rane, R. S. (2006), Pramana J. Phys. 67 (2): 237.

[29] Khadekar, G.S. and Tade, S. D. (2007), Astrophys. Space Sci., 310: 47.https://doi.org/10.1007/s10509-007-9410-2.

[30] Borkar, M. S. and Charjan, S. S. (2009), IJAM 22 (3): 445.

[31] Borkar, M. S. and Charjan, S. S. (2010a), J. Ind. Acad. Math 32(1): 147.

[32] Borkar, M. S. and Charjan, S. S. (2010b), an Int. J. AAM 5(1): 96.

[33] Borkar, M. S. and Charjan, S. S. (2010c), an Int. J. AAM 5(2): 563.
[34] Borkar, M. S., et al. (2013), Int. J. Appl. Math. 28(2): 1249.

[35] Borkar, M. S. and Charjan, S. S. (2013), an Int. J. AAM 8(1): 116.

[36] Borkar, M. S. and Gayakwad, P.V. (2013), American J. of Pure Appl. Mathematics 2(1):1. https://doi.org/10.11648/j.pamj.20130201.11.

[37] Borkar, M. S. and Gaikwad, N. P. (2014), Int. J. of Mathematical Sciences, 34 (1): 1406.

[38] Borkar, M. S. and Gayakwad, P. V. (2014a), Int. Org. of Scientific Res. J. of Math., 10(3): 28.

[39] Borkar, M. S. and Gayakwad, P.V. (2014b), an Int. J. AAM 9(1): 260.

[40] Borkar, M. S. and Ameen, A.(2015a), IJMPD 24 (2):1550019. https://doi.org/10.1142/S0218271815500194.

[41] Borkar, M. S. and Ameen, A. (2015b), the African Review of phys. 10. 0053: 443 .

[42] Borkar, M. S. and Ameen, A. (2017), Astrophysics 60 (2): 242 https://doi.org/10.1007/s10511-017-9479-4.

[43] Gaikwad, N. P., et al. (2011), Chin. Phys. Lett. 28(8): 089803. https://doi.org/10.1088/0256-307X/28/8/089803.

[44] Riess, A.G., et al. (2004), Astrophys. J. 607: 665. https://doi.org/10.1086/383612.

[45] Virey, J. M., et al. (2005), Phys. Rev. D, 72: 061302.https://doi.org/10.1103/PhysRevD.72.061302.

[46] Cunha, C.E., et al. (2009), Mon. Not. R. Astron. Soc., 396: 2379. https://doi.org/10.1111/j.1365-2966.2009.14908.x.

[47] Tripp, R. (1997), Astron.Astrophys.325: 871.

[48] Sahni, V. (1999), Pramana. 53: 937. https://doi.org/10.1007/s12043-999-0048-1.

[49] Tolman, R. C. (1934), Relativity, Thermodynamics and Cosmology (Oxford University Press, Oxford.

[50] Usmani, A.A., et al. (2008), Mon. Not. R. Astron. Soc., 386: L92.https://doi.org/10.1111/j.1745-3933.2008.00468.x.

[51] Ray, S., et al. (2011), Int J Theor Phys, 50 (9): 2687. https://doi.org/10.1007/s10773-011-0766-2.

[52] Katore, S. D., et al. (2011), Int J Theor Phys, 50 (8): 2477.https://doi.org/10.1007/s10773-011-0736-8. 